\title{
Implementation and Evaluation of Antimicrobial Stewardship Program in Medical ICU in Cairo University Specialized Pediatric Hospital
}

\author{
Mona Abdel Aziz Wassef ${ }^{1}$, Amal Mohamed Sayed ${ }^{1}$, Heba Sherif Abdel Aziz ${ }^{1 *}$, Bassant Meligy ${ }^{2}$, Mona Mohiedden Abdel Halim \\ ${ }^{1}$ Department of Clinical and Chemical Pathology, Faculty of Medicine, Cairo University, Cairo, Egypt; ${ }^{2}$ Department of Pediatric, \\ Faculty of Medicine, Cairo University, Cairo, Egypt
}

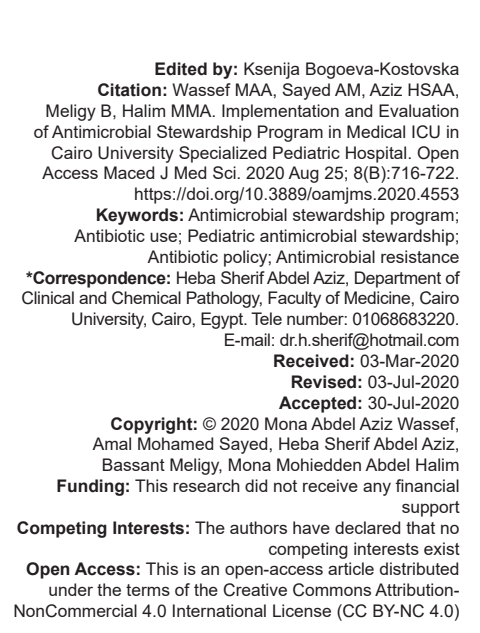

Introduction

Antibiotics abuse in the pediatric intensive care unit (PICU) is very high [1]. About one-third of prescribed antibiotics are inappropriate resulting in antibiotic resistance, especially with lacking new antibiotics. Hence, the importance of antimicrobial stewardship arises which means judicious and optimized use of antibiotics and reducing the adverse effect of antibiotics [2].

General practitioners in primary care settings are common causes of antibiotic overprescription, which has been shown to increase the risk of reattendance and medicalization of self-limiting infectious diseases [3].

The utilization of antibiotics is not restricted only in health care settings. Agriculture, aquaculture, and farming consume a lot of antibiotics. The higher the antibiotic use, the higher the rate of resistance [4].

In 2014, the Centre for Disease Control and Prevention developed the core elements of a successful hospital Antimicrobial Stewardship Program (ASP) [5].
World Health Organization has reported that infections caused by resistant organisms are not only detected in developing countries but throughout the world [6].

\section{Aim of the work}

The aim of the study was to set clinical practice guidelines customized according to local epidemiology of diseases and local cumulative antimicrobial susceptibility and assess ASP implementation in the PICU.

\section{Materials and Methods}

This prospective study was conducted at the (PICU) in Specialized Pediatric Hospital, Cairo University (CUSPH), from April 1, 2016, to June 30, 2017; pre-implementation phase from April 1, 2016, to 
September 30, 2016, intervention phase from October 1 , 2016, to December 31, 2016, and post-implementation phase from January 1, 2017, to June 30, 2017.

The study was performed in the clinical microbiology department in collaboration with the departments of pediatrics and pharmacy at the specialized pediatric hospital, Cairo University (CUSPH).

\section{Ethical approval}

The study was ethically approved by the research ethics committee of the Faculty of Medicine, Cairo University.

\section{Settings}

This prospective study was conducted at PICU with 12 beds capacity (6 beds per room) and 350 admissions per year in CUSPH which is a tertiary care teaching hospital. Staff of the unit working in 1:1 care (1 nurse/1 patient) in unstable patients and 1:2 care (1 nurse/2 patients) in stable non-ventilated patients. All admissions during the period of each phase were included within the corresponding phase.

\section{Flow of work}

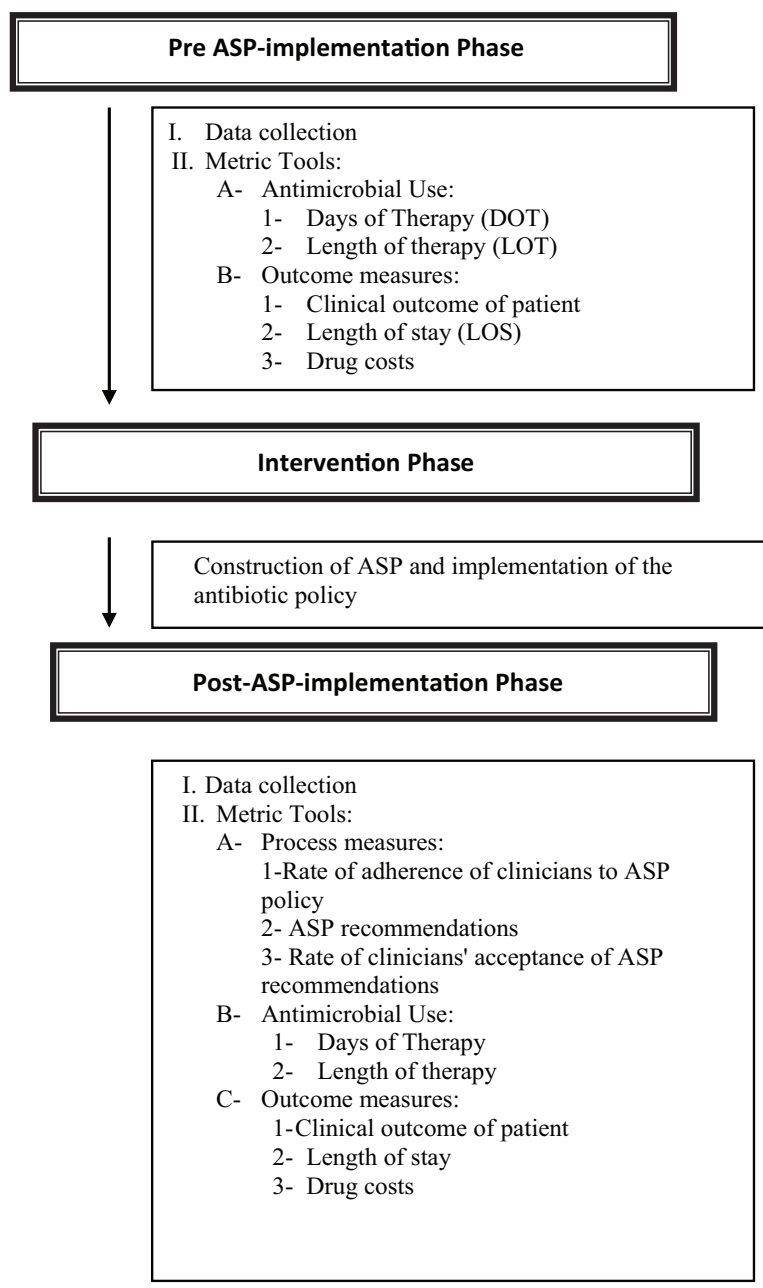

Data collection included name, age, sex, weight, date of admission and discharge, pediatric risk of mortality score (PRISM III score), clinical diagnosis and outcome of the patient, and indication and proof of infection at the start of antibiotic therapy, $48 \mathrm{~h}$ and 5 days reviews.

\section{Construction of ASP}

Multidisciplinary team members and their job description

- $\quad$ Team leader: Organized and communicated all team members with the management to obtain adequate authority and was responsible for the expected outcomes of the ASP

- $\quad$ Physician: Mainstay of stewardship program implementation

- Clinical pharmacists: Responsible for pharmacy-driven interventions

Clinical microbiologists: Provide clinical advice about microbiological investigations and construction of local cumulative annual antibiograms

- Infection control personnel: Monitored the healthcare-associated infections, antimicrobial resistance, and measures to prevent them

- Nurses: Implemented all elements of the ASP.

Before program implementation, the antimicrobial stewardship strategic plans were presented and approved by the ASP team members and the head of the studied PICU, to ensure their acceptance. The support and collaboration of hospital administration were essential for the success of the program

The ASP team members regularly met twice/ month to set policies that were applied in all situations for an optimal antibiotic prescription through:

Documentation of dose, duration, and indication for all courses of antibiotics to be easily identifiable and accessible

- Development and implementation of facility-specific treatment policy based on national guidelines and local antimicrobial susceptibilities

- Reassessment of continuous need and choice of empiric antibiotics after $48 \mathrm{~h}$ of initiating antibiotics when more diagnostic information was available (antibiotic time outs)

- Regular education and training of staff groups on antibiotics use in the form of presentations in formal and informal settings and electronic communication

PICU-specific antibiogram was constructed annually by microbiology laboratory staff members. 
After approval of the new guidelines by the ASP team, they were distributed to all PICU staff members to be ready for implementation.

The program was officially launched in April 1, 2016 as the first ASP at PICU of CUSPH.

\section{ASP implementation}

\section{Prior antimicrobial authorization}

The clinician requested the ASP team for each targeted antimicrobial agent using prior authorization form. The ASP team reviewed cultures and sensitivities and the regimen of treatment. The ASP pre-approved certain indications for selected targeted antimicrobial agents. Approval for empiric use of certain antimicrobial agents was usually given for $48 \mathrm{~h}$ until the availability of the results of the cultures.

The antimicrobial prescription was classified into three groups according to the need of culture and prior approval as shown in Table 1:

Table 1: Antimicrobial prescription classification

\begin{tabular}{lll}
\hline $\begin{array}{l}\text { Antimicrobials not requiring } \\
\text { culture or prior approval }\end{array}$ & $\begin{array}{l}\text { Antimicrobials requiring prior } \\
\text { approval without culture }\end{array}$ & $\begin{array}{l}\text { Antimicrobials requiring cultures } \\
\text { and prior approval }\end{array}$ \\
\hline Amoxicillin/flucloxacillin & Acyclovir & Maxipime \\
Amoxicillin/clavulanic acid & Azithromycin & Meropenem \\
Ampicillin/sulbactam & Cefobid & Imipenem \\
Amikacin & Ceftazidime & Ertapenem \\
Gentamicin & Ceftriaxone & Ciprofloxacin \\
& Cefotaxime & Levofloxacin \\
& Cefoperazone/sulbactam & Tigecycline \\
& Piperacillin/tazobactam & Colistin \\
& Amphotericin B & Vancomycin \\
& Fluconazole & Teicoplanin \\
& & Linezolid \\
& & Caspofungin \\
& & N.B.: Vancomycin, meropenem, \\
& & and imipenem only dispensed \\
& empirically in case of sepsis \\
\hline
\end{tabular}

\section{Metric tools}

Antimicrobial use measures

\section{DOT}

Was measured by calculating any amount of a specific antimicrobial agent administered on a calendar day to a particular patient, as documented in the medication administration record. The day of admission, discharge, and transfer to and from locations was included in the day's present count [7]

\section{LOT}

Was measured by calculating the number of days that a patient received systemic antimicrobials irrespective of their number and the total LOT of patients present during each phase was summed up [8].

\section{Process measures}

1. Rate of adherence of clinicians to ASP policy: Was measured by calculating the percent of patients treated according to the ASP policy to all patients admitted

2. ASP recommendations: Was measured by calculatingthepercentofeachASPrecommendation given to all ASP recommendations ASP recommendations: Dose increase, decrease; drug change; drug formulation change; schedule/frequency change; discontinuation; add medication; other changes to therapy; education/ counseling session; change diluents; change final solution concentration; change infusion rate; laboratory monitoring; request other investigation; request cultures; or no recommendation [9].

3. Rate of clinicians' acceptance to ASP recommendations: Was measured by calculating the percent of clinicians' acceptance to implement the ASP recommendation to all ASP given recommendations.

\section{Outcome measures}

1. Clinical outcome of patients received ASP recommendations: Was measured by calculating the percent of discharge and deaths among patients received treatment according to the ASP recommendations

2. LOS was measured by dividing the total number of occupied hospital bed-days by the total number of admissions or discharges [10]

3. Drug costs were measured based on summing up the monthly dispensed amount of each antimicrobial during the study period [8].

\section{Results}

One hundred fifty-one patients were admitted to the PICU in the pre-implementation phase; $55.3 \%$ of them were males with a mean age of $32.12 \pm 17.5$ months old. Moreover, in the post-implementation phase, 161 patients were admitted, $54.7 \%$ were males with a mean age of $24.53 \pm 12$ months old. The most common indication for antimicrobials in both phases was community-acquired pneumonia ( $45.3 \%$ and $58.3 \%$, respectively). The percent of patients who received prophylactic antimicrobials significantly decreased from $25.2 \%$ pre-implementation to $10 \%$ post-implementation $(p=0.002)$.

\section{Process measures results}

1. The rate of adherence of clinicians to ASP policy was $91.9 \%$

2. ASP recommendations

In the post-implementation phase, the most frequent antimicrobials required interventions were ampicillin-sulbactam $(21.6 \%)$ followed 


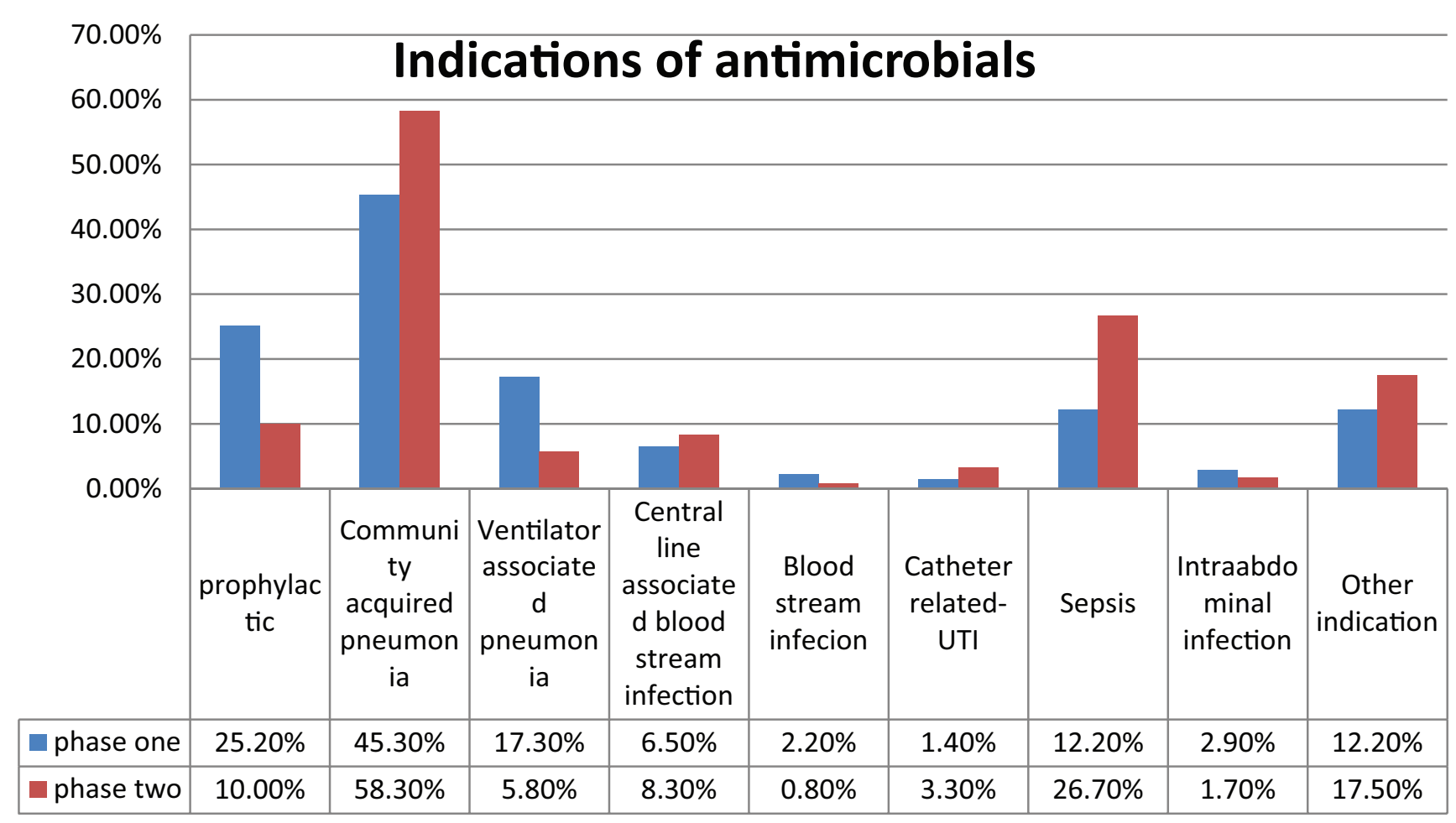

by imipenem (8.1\%) and vancomycin (6.4\%). The most frequent drug-related problem was dose calculation error (15.8\%) followed by prescribing an unnecessary drug with no medical indication (13.5\%). Moreover, the most frequent recommendation was drug schedule/ frequency change $(26.1 \%)$ followed by drug discontinuation (17.8\%).

3. The rate of acceptance to ASP recommendations among patients in the postimplementation phase was $93.7 \%$.

\section{Antibiotic use measures}

DOT

DOT of antibacterial drugs as shown in table 2 and DOT of all antimicrobials (antibacterial, antiviral, and antifungal) as shown in Table 3.

\section{LOT}

Significantly decreased from total 1502 days with mean 9.82 days pre-implementation to total 1318 days with mean 7.75 days post-implementation $(p=0.001)$.

\section{Outcome measures results}

Clinical outcome of patients

The percent of discharged patients was $68.9 \%$ pre-implementation and increased to $75.2 \%$ post-implementation. While the percent of deaths was $31.1 \%$ pre-implementation and decreased to $24.8 \%$ post-implementation $(p=0.203)$.

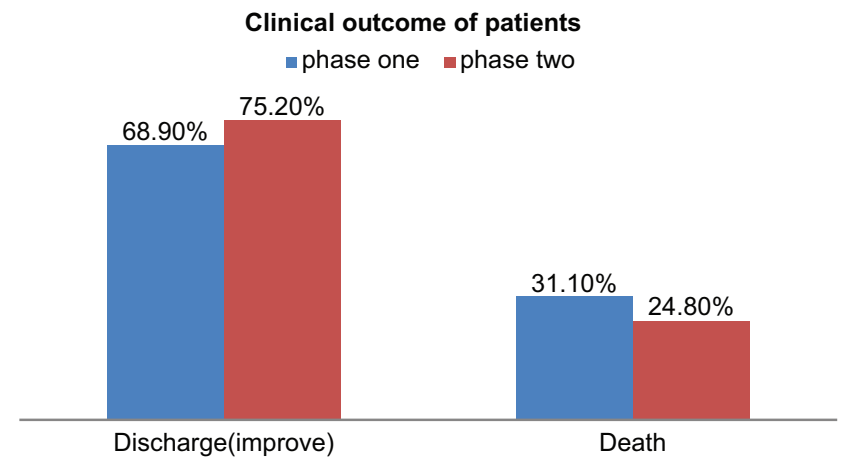

LOS

The mean \pm SD LOS was $10.66 \pm 6.00$ days pre-implementation and significantly decreased to 9.16 \pm 5.00 days post-implementation $(p=0.047)$.

Drug costs

Drug costs significantly decreased by $19.66 \%$ from (68164.94 L.E.) pre-implementation to (54764.96 L.E.) post-implementation $(p=0.01)$.

\section{Discussion}

ASP implementation in hospitals is mandatory, especially with steadily developing global antimicrobial resistance. The current study showed that the clinical 
services most commonly interacting with the ASP post-implementation were pediatric respiratory service $(40.4 \%)$ followed by neurological service (18.6\%), while the clinical service most commonly interacting with the ASP in a study of Newland et al., 2012 was general pediatric/resident service (20\%) followed by hematology/oncology (17\%) and hospitalist (17\%) [11].

The most common indication of antimicrobial intake was CAP (58.3\%) followed by sepsis $(26.7 \%)$ post-implementation. Newland et al., 2012 reported that the primary indications for antimicrobials included suspected sepsis (28\%), fever, neutropenia (12\%), and intra-abdominal infections (9\%) [11].

The percent of prophylactic antimicrobial courses in our study decreased significantly post-implementation from $25.2 \%$ to $10 \%$ ( $p<0.05$ ), and this was the aim of the study to optimize antibiotics use to decrease antibiotics expenditure. This result agreed with the study of Stocker et al., 2012 who reported that the percent of prophylactic antimicrobial courses decreased significantly post-implementation from $66.5 \%$ to $55.5 \%(p=0.02)$ [12].

Unlike our results, ASP implementation at PICU in Agha Khan University Hospital showed that the rate of antimicrobial courses taken for prophylaxis did not change in the pre-implementation phase $43 \%$ versus the post-implementation phase $43.2 \%$ [1].

The most common ASP recommendation in this study was drug schedule/frequency change (26.1\%) followed by drug discontinuation (17.8\%) which are consistent with those reported by Di Pentima et al., 2011 as the dose adjustment formed $40.2 \%$ of total recommendations ( 672 of 1673) while the modification of antimicrobial therapy formed $34.8 \%$ (583 of 1673) [13]. Other authors showed different recommendations; the most common was the modification of antimicrobial therapy which accounted for $48.5 \%$ in the study of Kreitmeyr et al., 2017, while in McCulloh et al. 2015 study, it was the discontinuation of antibiotic (28.6\%) and the most common antibiotic required recommendation was ceftriaxone (44\%) [2], [14].

The most frequent antimicrobial required interventions in our study were ampicillin-sulbactam $(21.6 \%)$ followed by imipenem $(8.1 \%)$ and vancomycin $(6.4 \%)$. Other studies, in the USA, showed different results, in Di Pentima et al., 2011 study the most frequent antimicrobial required interventions were vancomycin (16\%) (268 of 1673), piperacillin-tazobactam (11\%) (184 of 1673), ceftriaxone (6.3\%) (105 of 1673), and fluconazole (5\%) (82 of 1673) [13]. Newland et al., 2012 reported that the most common antimicrobials reviewed by the ASP included ceftriaxone/cefotaxime (43\%), vancomycin (18\%), and ceftazidime $(17 \%)$ of total 2378 recommendations [11].

The rate of acceptance of ASP recommendations in our study was $93.7 \%$ among 349 recommendations indicating the raised awareness of clinicians toward the rationalized antibiotics use in this tertiary hospital with the high flow of patients and that was slightly higher than that reported by DiPentima etal., 2011 and McCulloh etal., 2015 which was $86.9 \%$ in both studies (total recommendations were 1637, 350 respectively), [13], [14] and it was $80 \%$ of total recommendations 2378 in a study of Newland et al., 2012 [11].

The rate of acceptance of ASP recommendations in Kreitmeyr et al., 2017 study in German hospitals was slightly higher than in our study $(95.8 \%)$ of total recommendations 167 [2].

The percent of patients received antimicrobials was $92.7 \%$ pre-implementation (151 patients) compared to $74.5 \%$ post-implementation (161 patients) $(p<0.001)$ indicating the beneficial effect of ASP in optimizing antibiotic use. Unlike our results, the impact of ASP in Kreitmeyr et al., 2017 study which revealed a minimal change in the percent of hospitalized children who received antimicrobials pre-implementation (30.6\%) (1007 patients) versus post-implementation (30.5\%) (967 patients) [2].

Our study showed the positive effect of the ASP in optimizing antibiotic use as all antibiotics; DOT decreased by $13.5 \%$, with significant effect regarding ceftazidime, ceftriaxone, and amikacin. Other antibiotics DOT, including cephalosporins, aminopenicillins with beta-lactamase (ampicillin-sulbactam and amoxicillinclavulanic acid) piperacillin-tazobactam, meropenem, ciprofloxacin, all aminoglycosides, trimethoprimsulfamethoxazole, vancomycin, and metronidazole also showed reduction post-implementation (Table 2). Kreitmeyr et al. 2017 study showed a similar reduction in all antibiotic use by $10.5 \%$ post-implementation, this reduction was significant in $3^{\text {rd }}$ generation cephalosporins (22.3\%), fluoroquinolones (59.9\%) (from 32 to 13 DoT/1000 PD), and metronidazole (51.1\%) (from 27 to 13 DoT/1000 PD). However, they differ from our study in the consumption of combined aminopenicillins with beta-lactamase inhibitors as ampicillin-sulbactam and amoxicillin-clavulanic acid in which DOT significantly increased by $78.8 \%$ (from 17.1 DoT/1000 PD to 30.5 DoT/1000 PD) [2].

Other studies reported similar effective ASP in a significant reduction of all antibiotics DOT/1000 PD in the ASP period includes Haque et al. study which reported reduction by $64 \%$ from $3477 D O T / 1000$ PD to 1323DOT/1000 PD and Newland et al. study which reported reduction from 883 DOT/1000 PD to 787 DOT/1000 PD [1], [11].

This study showed significantly increased DOT in gentamicin by $75.4 \%$ (from 23.3DOT/1000 PD to 94.7 DOT/1000 PD ) ( $p=0.009)$, unlike Di Pentima et al., 2011 study which showed a significant reduction in the use of gentamicin (from 372 doses/1000 PD to 92 doses/1000 PD) $(p<0.001)$ [13].

Unlike our results where carbapenem DOT/1000 PD showed a mild insignificant increase by 
Table 2: DOT antibacterial drugs

\begin{tabular}{|c|c|c|c|c|c|c|c|c|c|}
\hline \multirow[t]{2}{*}{ Antibiotic } & \multicolumn{3}{|c|}{ Phase one - patient days $=1804$} & \multicolumn{3}{|c|}{ Phase two - patient days $=1648$} & \multicolumn{3}{|c|}{ Difference } \\
\hline & DOT total & DOT/1000PD & $\%$ & DOT total & DOT/1000PD & $\%$ & p-value & DOT/1000PD & $\%$ \\
\hline All $\left(3^{\text {rd }}+4^{\text {th }}\right)$ cephalosporins & 271 & 150.2 & 9.7 & 153 & 92.8 & 6.9 & 0.093 & -57.4 & -38.2 \\
\hline $3^{\text {rd }}$ cephalosporins & 267 & 148 & 9.5 & 122 & 74 & 5.5 & 0.065 & -74 & -50 \\
\hline Ceftazidime & 60 & 33.3 & 2.1 & 4 & 2.4 & 0.2 & 0.041 & -30.9 & -92.8 \\
\hline Ceftriaxone & 161 & 89.2 & 5.7 & 51 & 31 & 2.3 & 0.026 & -58.2 & -65.2 \\
\hline Cefotaxime & 46 & 25.5 & 1.6 & 67 & 40.7 & 3 & 0.937 & 15.2 & 37.3 \\
\hline $4^{\text {th }}$ cephalosporins & 4 & 2.2 & 0.1 & 31 & 18.8 & 1.4 & 0.310 & 16.6 & 88.3 \\
\hline All combinations & 605 & 335.4 & 21.6 & 380 & 230.6 & 17.1 & 0.180 & -104.8 & -31.2 \\
\hline Amoxicillin/clavulanic acid + Ampicillin/sulbactam & 424 & 235 & 15.1 & 292 & 177.2 & 13.2 & 0.394 & -57.8 & -24.6 \\
\hline Amoxicillin/clavulanic acid & 29 & 16.1 & 1 & 0 & 0 & 0 & 0.065 & -16.1 & \\
\hline Ampicillin/sulbactam & 395 & 219 & 14.1 & 292 & 177.2 & 13.2 & 0.485 & -41.8 & -19.1 \\
\hline Cefoperazone/sulbactam & 0 & 0 & 0 & 18 & 11 & 0.8 & 0.699 & 11 & \\
\hline Piperacillin/tazobactam & 181 & 100.3 & 6.4 & 70 & 42.5 & 3.2 & 0.180 & -57.8 & -57.6 \\
\hline All carbapenems & 407 & 225.6 & 14.5 & 427 & 259.1 & 19.2 & 0.485 & 33.5 & 1.4 \\
\hline Imipenem & 87 & 48.2 & 3.1 & 154 & 93.4 & 6.9 & 0.394 & 45.2 & 48.4 \\
\hline Meropenem & 320 & 177.4 & 11.4 & 239 & 145 & 10.8 & 0.485 & -32.4 & -18.3 \\
\hline Ertapenem & 0 & 0 & 0 & 34 & 20.6 & 1.5 & 0.180 & 20.6 & \\
\hline All fluoroquinolones & 262 & 145.2 & 9.3 & 306 & 185.7 & 13.8 & 0.065 & 40.5 & 21.8 \\
\hline Ciprofloxacin & 229 & 127 & 8.2 & 187 & 113.5 & 8.4 & 0.818 & -13.5 & -10.6 \\
\hline Levofloxacin & 33 & 18.3 & 1.2 & 119 & 72.2 & 5.4 & 0.026 & 53.9 & 74.7 \\
\hline All aminoglycosides & 443 & 245.6 & 15.8 & 270 & 163.8 & 12.2 & 0.485 & -81.8 & -33.3 \\
\hline Amikacin & 401 & 222.3 & 14.3 & 114 & 69.2 & 5.3 & 0.004 & -153.1 & -68.9 \\
\hline Gentamycin & 42 & 23.3 & 1.5 & 156 & 94.7 & 7 & 0.009 & 71.4 & 75.4 \\
\hline Trimethoprim/sulfamethoxazole & 116 & 64.3 & 4.1 & 31 & 18.8 & 1.4 & 0.240 & -45.5 & -70.8 \\
\hline Polymyxin B & 99 & 54.9 & 3.5 & 121 & 73.4 & 5.5 & 0.589 & 18.5 & 25.2 \\
\hline Tigecycline & 13 & 7.2 & 0.5 & 13 & 7.9 & 0.6 & 0.699 & 0.7 & 8.9 \\
\hline Neomycin & 7 & 3.9 & 0.2 & 31 & 18.8 & 1.4 & 0.589 & 14.9 & 79.3 \\
\hline Metronidazole & 122 & 67.6 & 4.3 & 70 & 42.5 & 3.2 & 0.485 & -25.1 & -37.1 \\
\hline Vancomycin & 349 & 193.6 & 12.4 & 179 & 108.6 & 8.1 & 0.132 & -85 & -43.9 \\
\hline Teicoplanin & 18 & 10 & 0.6 & 27 & 16.4 & 1.2 & 0.065 & 6.4 & 40.1 \\
\hline Erythromycin & 86 & 47.7 & 3.1 & 119 & 72.2 & 5.4 & 0.394 & 24.5 & 33.9 \\
\hline Clindamycin & 9 & 5 & 0.3 & 92 & 55.8 & 4.1 & 0.026 & 50.8 & 91 \\
\hline All antibiotics & 2807 & 1556 & 100 & 2219 & 1346.4 & 100 & 0.394 & -209.6 & -13.5 \\
\hline
\end{tabular}

Table 3: DOT - all antimicrobials (antibacterial, antiviral, and anti-fungal drugs)

\begin{tabular}{|c|c|c|c|c|c|c|c|c|c|}
\hline \multirow[t]{2}{*}{ Antimicrobial } & \multicolumn{3}{|c|}{ Phase one - patient days $=1804$} & \multicolumn{3}{|c|}{ Phase two - patient days $=1648$} & \multicolumn{3}{|c|}{ Difference } \\
\hline & DOT total & DOT/1000PD & $\%$ & DOT total & DOT/1000PD & $\%$ & $p$-value & DOT/1000PD & $\%$ \\
\hline All antibiotics & 2807 & 1556 & 88.1 & 2219 & 1346.4 & 90.1 & 0.394 & -209.6 & -13.5 \\
\hline Acyclovir & 100 & 55.4 & 3.1 & 149 & 29.7 & 6 & 0.394 & -25.7 & -46.4 \\
\hline Tamiflu & 8 & 4.4 & 0.3 & 9 & 5.5 & 0.4 & 0.818 & 1.1 & 20 \\
\hline Amphotericin B & 64 & 35.5 & 2 & 18 & 11 & 0.7 & 0.485 & -24.5 & -69 \\
\hline Fluconazole & 206 & 114.2 & 6.5 & 157 & 95.3 & 6.4 & 1.000 & -18.9 & -33.9 \\
\hline Mycamine & 0 & 0 & 0 & 12 & 7.3 & 0.5 & 0.394 & 7.3 & \\
\hline All antimicrobials & 3185 & 1765.5 & 100 & 2464 & 1495.1 & 100 & 0.180 & -270.4 & -15.3 \\
\hline
\end{tabular}

1.4\% (from 225.6 DOT/1000 PD to 259.1 DOT/1000 PD), Horikoshi et al., 2017 Japan reported that carbapenem DOT/1000 PD significantly decreased by $59.3 \%$ (from 4.94 DOT/1000 PD to 2.01 DOT/1000 PD) [15].

Certain antimicrobials consumption improved post-implementation, as the DOT of amphotericin B declined by $69 \%$ (from 35.5 DOT/ 1000 PD to 11 DOT/ 1000 PD) ( $p=0.485)$. Similarly, Di Pentima et al. 2011 reported that amphotericin $B$ was peaked at 50 doses pre-implementation versus 4 doses per 1000 patient days post-implementation ( $p<0.0001)$ [13].

A comparison in average antibiotic use was done in the USA ( 9 hospitals implemented ASP and 22 hospitals did not implement ASP). ASP+ hospital experienced a greater decline in all antibiotic use; $(11 \%)$ versus (8\%) in ASP- hospitals, $(p=0.04)$ [16].

The length of therapy in our study showed significant improvement which also denotes the positive effect of the ASP on antibiotic use (from 1502 days to 1318 days) ( $p=0.001$ ). Similarly, Kreitmeyr et al., 2017 study demonstrated a significant decline in overall LOT by $7.7 \%$ (from 377.4 to 348.3 LOT/1000 PD) $(p=0.02$ ) [2].

Similarly, the length of therapy decreased from 567 to 523 LOT per 1000 PD ( $p$ 0.001) in Newland et al., 2012 [11].
The overall mortality in the current study decreased insignificantly from $31.1 \%$ (among 151 patients) to $24.8 \%$ (among 161 patients). Similarly, Kreitmeyr et al., 2017 reported an insignificant difference regarding in-hospital mortality during pre- and postimplementation periods $(0.37 \%$ among 273 patients vs. $0.38 \%$ among 263 patients, respectively) [2]. However, Horikoshi et al. 2017 reported a significant decrease (from 0.087/1000 PD to 0.051/1000 PD) in infectionrelated mortality due to ASP's positive effect on the clinical outcome of the patient.

In this work, the mean length of hospital stays showed a significant decline from mean 10.66 days to 9.16 days post-implementation, giving this overcrowded tertiary hospital a better capacity to provide medical services for more patients. Comparable findings were reported by Horikoshi et al., 2017 (from mean 20.6 to 18.6 days), [15] while Kreitmeyr et al. 2017 reported that the average length of stay remained stable with a median 7 days (range 1-93 days) versus 6 days (range 2-123 days) [2].

Antimicrobial costs significantly reduced by $19.66 \%$ ( $p=0.01)$, which is consistent with the report of Haque et al., 2018, where the drug costs were significantly decreased by $58 \%(p<0.0001)$ [1] 


\section{Conclusion and Recommendations}

The ASP is very important and very effective in optimizing and improving the antibiotic use to reach the best clinical outcome of the patients and to decrease hospital length of stay and drug costs.

Considering the relatively small population study and the short period of observation, lack of financial resources, lack of effective IT electronic system linking the multidisciplinary departments together, we recommend ASP implementation over a larger population and hospital settings with more comprehensive administration involvement that could afford financial resources and commit all departments to the ASP.

\section{Ethical committee}

The study was ethically approved by the research ethics committee of the faculty of medicine, Cairo University.

\section{References}

1. Haque A, Hussain K, Ibrahim R, Abbas Q, Ahmed SA, Jurair H, et al. Impact of a pharmacist-led antibiotic stewardship program in a PICU of low/middle-income country. BMJ Open Qual. 2018;7(1):e000180. https://doi.org/10.1136/bmjoq-2017-000180 PMid:29333498

2. Kreitmeyr K, von Both U, Pecar A, Borde JP, Mikolajczyk R, Huebner J. Pediatric antibiotic stewardship: Successful interventions to reduce broad-spectrum antibiotic use on general pediatric wards. Infection. 2017;45(4):493-504. https:// doi.org/10.1007/s15010-017-1009-0

PMid:28397171

3. Llor C, Bjerrum L. Antimicrobial resistance: Risk associated with antibiotic overuse and initiatives to reduce the problem. Ther Adv Drug Saf. 2014;5(6):229-41. https://doi. org/10.1177/2042098614554919

PMid:25436105

4. Meek RW, Vyas H, Piddock LJ. Nonmedical uses of antibiotics: Time to restrict their use? PLoS Biol. 2015;13(10):e1002266. https://doi.org/10.1371/journal.pbio.1002266

PMid:26444324

5. Centers for Disease Control and Prevention. Core Elements of Hospital Antibiotic Stewardship Programs. Atlanta: Centers for Disease Control and Prevention; 2014. https://doi.org/10.1093/

\section{cid/ciu542}

6. Exner M, Bhattacharya S, Christiansen B, Gebel J, GoroncyBermes P, Hartemann P, et al. Antibiotic resistance: What is so special about multidrug-resistant Gram-negative bacteria? GMS Hyg Infect Control. 2017;12:Doc05.

PMid:28451516

7. Centers for Disease Control and Prevention. Antimicrobial Use and Resistance (AUR) Module. Atlanta: Centers for Disease Control and Prevention; 2017

8. Morris AM, Brener S, Dresser L, Daneman N, Dellit TH, Avdic E, et al. Use of a structured panel process to define quality metrics for antimicrobial stewardship programs. Infect Control Hosp Epidemiol. 2012;33(5):500-6. https://doi.org/10.1086/665324 PMid:22476277

9. van Mil JW, Horvat N, Westerlund T. Classification for DrugRelated Problems V7.0 the PCNE Classification V7.0, 20032016 Pharmaceutical Care Network Europe Foundation; 2016.

10. World Health Organization. Average Length of Stay. Geneva: World Health Organization; 2018. Available from: https://www. gateway.euro.who.int/en/indicators/hfa_540-6100-averagelength-of-stay-all-hospitals. [Last accessed on 2019 Jan 05]. https://doi.org/10.1787/888933155611

11. Newland JG, Stach LM, De Lurgio SA, Hedican E, Yu D, Herigon JC, et al. Impact of a prospective-audit-with-feedback antimicrobial stewardship program at a children's hospital. J Pediatric Infect Dis Soc. 2012;1(3):179-86. https://doi. org/10.1093/jpids/pis054

PMid:26619405

12. Stocker M, Ferrao E, Banya W, Cheong J, Macrae D, Furck A Antibiotic surveillance on a paediatric intensive care unit: Easy attainable strategy at low costs and resources. BMC Pediatr. 2012;12:196. https://doi.org/10.1186/1471-2431-12-196 PMid:23259701

13. Di Pentima MC, Chan S, Hossain J. Benefits of a pediatric antimicrobial stewardship program at a children's hospital. Pediatrics. 2011;128(6):1062-70. https://doi.org/10.1542/ peds.2010-3589 PMid:22106075

14. McCulloh RJ, Queen MA, Lee B, Yu D, Stach L, Goldman J, et al. Clinical impact of an antimicrobial stewardship program on pediatric hospitalist practice, a 5-year retrospective analysis. Hosp Pediatr. 2015;5(10):520-7. https://doi.org/10.1542/ hpeds.2014-0250 PMid:26427920

15. Horikoshi $\mathrm{Y}$, Suwa J, Higuchi H, Kaneko T, Furuichi M, Aizawa $\mathrm{Y}$, et al. Sustained pediatric antimicrobial stewardship program with consultation to infectious diseases reduced carbapenem resistance and infection-related mortality. Int J Infect Dis. 2017;64:69-73. https://doi.org/10.1016/j.jijid.2017.09.012 PMid:28941633

16. Hersh AL, De Lurgio SA, Thurm C, Lee BR, Weissman SJ, Courter JD, et al. Antimicrobial stewardship programs in freestanding children's hospitals. Pediatrics. 2015;135(1):33-9. https://doi.org/10.1542/peds.2014-2579

PMid:25489018 